\title{
Mass conserving global solutions for the nonlinear collision-induced fragmentation model with a singular kernel
}

\author{
Debdulal Ghosh ${ }^{1}$, Jayanta Paul ${ }^{1}$, and Jitendra Kumar ${ }^{1}$ \\ ${ }^{1}$ Indian Institute of Technology Kharagpur
}

January 31, 2022

\begin{abstract}
This article is devoted to the study of existence of a mass conserving global solution for the collision-induced nonlinear fragmentation model which arises in particulate processes, with the following type of collision kernel: $\backslash\left[\mathrm{C}(\mathrm{x}, \mathrm{y})^{\sim} \backslash \mathrm{le}^{\sim} \mathrm{k}_{-} 1 \backslash\right.$ frac $\{(1+$ $\left.\mathrm{x})^{\wedge} \backslash \mathrm{nu}(1+\mathrm{y})^{\wedge} \backslash \mathrm{nu}\right\}\left\{\backslash \operatorname{left}(\mathrm{xy} \backslash \text { right })^{\wedge} \backslash\right.$ sigma $\left.\}, \backslash\right]$ for all $\sim$ x, y $\backslash$ in $(0, \backslash$ infty $) \$$, where $\$ \mathrm{k} \_1 \$$ is a positive constant, $\$ \backslash$ sigma $\backslash$ in $\backslash$ left $[0, \backslash \operatorname{tfrac}\{1\}\{2\} \backslash$ right $] \$$ and $\$ \backslash$ nu $\backslash$ in $[0,1] \$$. The above-mentioned form includes many practical oriented kernels of both $\backslash$ emph\{singular\} and $\backslash \operatorname{emph}\{$ non-singular\} types. The singularity of the unbounded collision kernel at coordinate axes extends the previous existence result of Paul and Kumar [Mathematical Methods in the Applied Sciences 41 (7) (2018) 2715-2732 ( $\backslash$ href $\{$ https://doi.org/10.1002/mma.4775\}\{doi:10.1002/mma.4775\})] and also exhibits at most quadratic growth at infinity. Finally, uniqueness of solution is also investigated for pure singular collision rate, i.e., for $\sim \$ \backslash n u=0 \$$.
\end{abstract}

\section{Hosted file}

Collisional_singular.pdf available at https://authorea.com/users/424877/articles/554772mass-conserving-global-solutions-for-the-nonlinear-collision-induced-fragmentationmodel-with-a-singular-kernel 